\title{
Multiple alignment of membrane proteins for measuring residual dipolar couplings using lanthanide ions bound to a small metal chelator
}

Douglas E. Kamen, Sean M. Cahill, and Mark E. Girvin

Biochemistry Department, Albert Einstein College of Medicine, 1300 Morris Park Ave, Bronx NY, 10461

Supporting Information

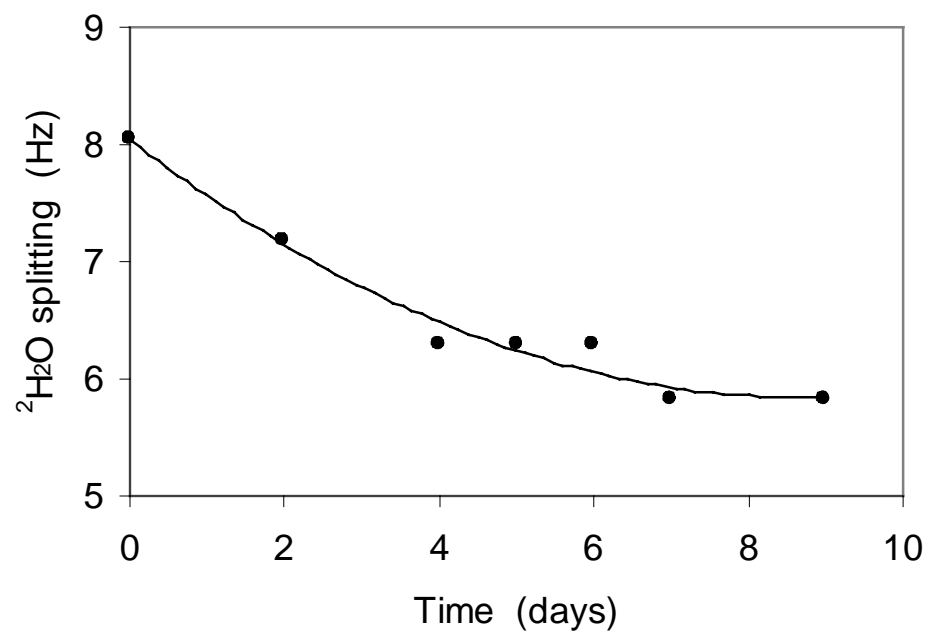

Figure S1. Decrease in deuterium splitting over time. Measurements were made at $47^{\circ} \mathrm{C}$ on a Bruker DRX 600. Sample conditions were $6 \%$ polyacrylamide, $50 \mathrm{mM}$ potassium phosphate, $5 \%$ LPPG, $1 \mathrm{mM}$ subunit c, pH 6.8. The curve is a simple trendline. 

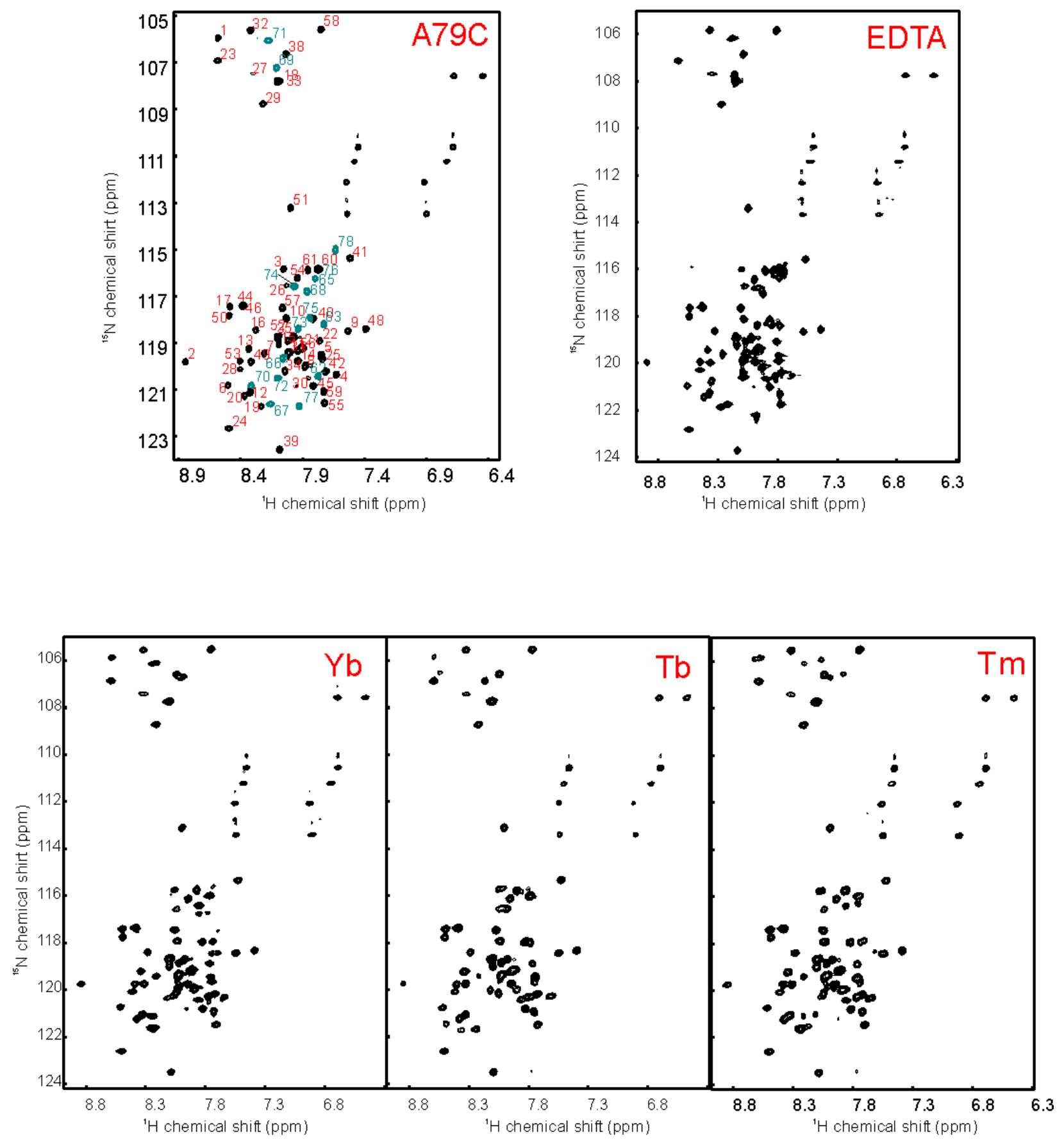

Figure S2. HSQC spectra of A79C mutant subunit c. Clockwise from top left are unmodified A79C, A79C with EDTA modification and no bound metal, and modified with $\mathrm{Tm}^{3+}, \mathrm{Tb}^{3+}$, or $\mathrm{Yb}^{3+}$. Assignments are shown in the first panel, with red labels indicating resonances that do not shift on addition of lanthanide, and green identifying those that do. 
Table S1. Measured ${ }^{1} \mathrm{H}^{15} \mathrm{~N}$ and ${ }^{13} \mathrm{C}^{, 13} \mathrm{C}^{\alpha} \mathrm{RDC}$ values for $\mathrm{A} 79 \mathrm{C}$ with bound lanthanide ions.

\begin{tabular}{|c|c|c|c|c|c|c|}
\hline \multirow[t]{2}{*}{ Residue } & \multicolumn{2}{|c|}{$\mathrm{Tm}^{3+}$ at $800 \mathrm{MHz}$} & \multicolumn{2}{|c|}{$\mathrm{Yb}^{3+}$ at $800 \mathrm{MHz}$} & \multicolumn{2}{|c|}{$\mathrm{Tb}^{3+}$ at $900 \mathrm{MHz}$} \\
\hline & $\mathrm{HN}$ & $C^{\prime} C^{\alpha}$ & $\mathrm{HN}$ & $\mathrm{C}^{\prime} \mathrm{C}^{\alpha}$ & $\mathrm{HN}$ & $C^{\prime} C^{\alpha}$ \\
\hline 1 & & -0.2 & & -3.9 & & 1.6 \\
\hline 2 & 3.4 & 1.3 & 0.3 & 0.1 & 2.0 & -0.1 \\
\hline 3 & -2.3 & 1.7 & 2.2 & 0.9 & -4.1 & 1.1 \\
\hline 4 & -1.1 & -3.8 & -1.3 & 0.2 & 0.4 & 1.8 \\
\hline 5 & -0.1 & 2.6 & 2.0 & 0.4 & 2.4 & 0.9 \\
\hline 6 & -1.0 & -0.6 & 2.0 & -0.4 & 0.1 & -3.4 \\
\hline 7 & 0.6 & -7.3 & -0.6 & 0.0 & 0.7 & 1.1 \\
\hline 8 & -4.6 & -1.8 & -0.1 & 0.1 & -5.0 & 0.8 \\
\hline 9 & -0.7 & -0.5 & -1.1 & 1.9 & 2.9 & 2.2 \\
\hline 10 & 2.8 & 8.9 & -3.4 & 4.4 & 5.9 & 3.0 \\
\hline 11 & -8.3 & 0.8 & n.d. & 2.1 & n.d. & 2.8 \\
\hline 12 & -6.4 & 0.9 & -1.5 & -0.1 & -1.4 & -4.3 \\
\hline 13 & -0.6 & -1.1 & -1.6 & 1.0 & -3.1 & 2.3 \\
\hline 14 & -5.7 & n.d. & 2.3 & n.d. & -0.8 & n.d. \\
\hline 15 & 6.1 & -5.2 & -2.4 & -0.4 & -0.2 & 1.8 \\
\hline 16 & 2.7 & 3.9 & 2.8 & -0.8 & -0.8 & 3.6 \\
\hline 17 & -1.5 & 3.1 & -0.4 & 0.5 & 3.3 & 8.6 \\
\hline 18 & 3.7 & -2.9 & 4.3 & -5.7 & -2.0 & -6.0 \\
\hline 19 & 0.7 & 1.7 & 4.7 & 0.0 & 0.1 & 1.2 \\
\hline 20 & -1.8 & 3.4 & -1.2 & 3.1 & 1.9 & -4.1 \\
\hline 21 & -1.2 & n.d. & 5.1 & n.d. & 2.8 & -0.6 \\
\hline 22 & -5.2 & 0.7 & 2.4 & 0.3 & -6.8 & 5.8 \\
\hline 23 & 3.9 & -1.9 & 0.5 & -0.5 & 2.7 & -1.7 \\
\hline 24 & -2.3 & n.d. & 2.6 & n.d. & -1.5 & n.d. \\
\hline 25 & 5.7 & n.d. & -0.3 & n.d. & 1.8 & n.d. \\
\hline 26 & -10.6 & n.d. & -3.8 & -2.2 & -8.1 & n.d. \\
\hline 27 & 5.0 & -6.3 & n.d. & -1.7 & 0.0 & -8.2 \\
\hline 28 & -2.0 & -0.9 & 2.3 & -0.5 & 3.5 & -4.3 \\
\hline 29 & -0.1 & -1.8 & 5.2 & 3.3 & 0.1 & 5.2 \\
\hline 30 & -1.5 & -2.0 & -1.2 & 3.7 & -2.7 & -5.6 \\
\hline 31 & -1.9 & -2.0 & 2.2 & 0.2 & 1.6 & -3.9 \\
\hline 32 & 1.3 & 0.9 & 2.4 & -0.5 & 1.9 & -2.3 \\
\hline 33 & 2.6 & 1.0 & 1.8 & -0.2 & 3.5 & 1.3 \\
\hline 34 & -2.4 & -0.1 & 0.9 & -0.4 & -4.0 & 1.5 \\
\hline 35 & -0.1 & 1.9 & -5.1 & 1.0 & -1.0 & 0.1 \\
\hline 36 & 1.3 & 2.3 & 0.8 & 0.4 & -1.8 & 0.7 \\
\hline 37 & 0.9 & 0.9 & -0.4 & 0.4 & 0.5 & -0.5 \\
\hline 38 & 1.4 & -0.7 & 5.3 & -0.3 & 3.5 & -4.5 \\
\hline 39 & -4.1 & -0.4 & -2.4 & -0.1 & -2.2 & 0.0 \\
\hline 40 & 1.2 & -1.3 & -0.3 & 0.3 & 1.3 & -1.8 \\
\hline 41 & -0.6 & 1.6 & 0.5 & 0.3 & -2.8 & -1.2 \\
\hline 42 & 1.8 & n.d.p. & 2.8 & n.d.p. & -2.4 & n.d.p. \\
\hline 43 & n.d.p. & -0.9 & n.d.p. & 0.1 & n.d.p. & 1.5 \\
\hline 44 & -0.8 & -0.7 & -1.1 & 0.4 & 3.0 & -0.5 \\
\hline 45 & 0.1 & 4.0 & -2.0 & 1.7 & 0.0 & 3.3 \\
\hline 46 & -0.8 & n.d.p. & 3.3 & n.d.p. & -3.2 & n.d.p. \\
\hline 47 & n.d.p. & 2.6 & n.d.p. & 0.3 & n.d.p. & -0.9 \\
\hline 48 & 0.7 & -1.8 & 2.4 & 0.7 & -1.3 & -4.2 \\
\hline 49 & -1.8 & -2.5 & -1.0 & -1.8 & 1.8 & -1.8 \\
\hline
\end{tabular}




$\begin{array}{rrrrrrr}50 & 1.7 & 0.6 & 2.4 & 0.5 & 5.1 & 0.3 \\ 51 & 0.1 & -7.4 & 3.0 & 0.3 & -0.6 & -1.0 \\ 52 & -2.5 & -1.4 & 0.0 & -1.3 & 2.7 & -1.8 \\ 53 & -2.5 & 3.5 & 2.8 & 2.4 & -1.0 & -5.2 \\ 54 & -0.3 & -2.2 & -2.4 & 0.8 & 1.3 & 1.4 \\ 55 & -5.3 & 2.2 & -3.3 & 3.0 & -1.6 & -0.3 \\ 56 & 3.1 & 0.6 & 0.1 & -0.3 & 1.2 & -1.8 \\ 57 & -2.4 & 0.7 & -0.8 & -0.4 & -2.7 & 1.1 \\ 58 & 2.8 & -0.9 & -1.6 & -0.2 & 1.5 & -4.0 \\ 59 & -2.9 & -2.4 & -6.6 & -1.2 & -0.8 & -1.5 \\ 60 & -4.1 & 0.6 & -3.5 & 1.1 & -2.1 & 3.5 \\ 61 & -2.5 & 0.6 & -2.1 & 0.4 & -1.6 & -8.9 \\ 62 & 4.4 & -5.6 & -1.9 & \text { n.d. } & 1.8 & 3.6 \\ 63 & 1.9 & \text { n.d.p. } & \text { n.d. } & \text { n.d.p. } & -5.7 & \text { n.d.p. } \\ 64 & \text { n.d.p. } & \text { n.d. } & \text { n.d.p. } & \text { n.d. } & \text { n.d.p. } & \text { n.d. } \\ 65 & \text { n.d. } & \text { n.d. } & \text { n.d. } & \text { n.d. } & \text { n.d. } & \text { n.d. } \\ 66 & \text { n.a. } & \text { n.a. } & 1.4 & \text { n.a. } & \text { n.a. } & 2.8 \\ 67 & 1.4 & \text { n.a. } & \text { n.a. } & \text { n.a. } & 0.5 & 4.8 \\ 68 & -2.1 & \text { n.a. } & -1.7 & \text { n.a. } & -1.2 & -1.9 \\ 69 & \text { n.a. } & \text { n.a. } & \text { n.a. } & \text { n.a. } & 2.0 & \text { n.a. } \\ 70 & \text { n.a. } & \text { n.a. } & \text { n.a. } & \text { n.a. } & \text { n.a. } & \text { n.a. } \\ 71 & \text { n.a. } & \text { n.a. } & \text { n.a. } & \text { n.a. } & \text { n.a. } & \text { n.a. } \\ 72 & \text { n.a. } & \text { n.a. } & \text { n.a. } & \text { n.a. } & \text { n.a. } & \text { n.a. } \\ 73 & \text { n.a. } & \text { n.a. } & \text { n.a. } & \text { n.a. } & \text { n.a. } & \text { n.a. } \\ 74 & \text { n.a. } & \text { n.a. } & \text { n.a. } & \text { n.a. } & \text { n.a. } & \text { n.a. } \\ 75 & \text { n.a. } & \text { n.a. } & \text { n.a. } & \text { n.a. } & \text { n.a. } & \text { n.a. } \\ 76 & \text { n.a. } & \text { n.a. } & \text { n.a. } & \text { n.a. } & \text { n.a. } & \text { n.a. } \\ 77 & \text { n.a. } & \text { n.a. } & \text { n.a. } & \text { n.a. } & \text { n.a. } & \text { n.a. } \\ 78 & \text { n.a. } & \text { n.a. } & \text { n.a. } & \text { n.a. } & \text { n.a. } & \text { n.a. } \\ 79 & \text { n.a. } & \text { n.a. } & \text { n.a. } & \text { n.a. } & \text { n.a. } & \text { n.a. } \\ & & & & & & \end{array}$

n.a. - Not assigned due to large pseudocontact shift.

n.d. - Not determined due to low signal to noise ratio.

n.d.p. - Not assigned due to proline. 


\section{Methods}

Preparation of $A 79 C$. The mutation was generated using the Quickchange Site Directed Mutagenesis Kit (Stratagene). Purification and sample preparation was carried out as described ${ }^{1-}$ ${ }^{3}$, with some modifications. Protein samples in $1: 1 \mathrm{CHCl}_{3}: \mathrm{CH}_{3} \mathrm{OH}$ with $15 \mathrm{mg}$ of LPPG were dried under a stream of argon. It was necessary to add DTT to 3-5 $\mathrm{mM}$ in order to prevent intermolecular disulfide bond formation.

(1) Girvin, M. E.; Fillingame, R. H. Biochemistry 1995, 34, 1635-1645.

(2) Girvin, M. E.; Rastogi, V. K.; Abildgaard, F.; Markley, J. L.; Fillingame, R. H. Biochemistry 1998, $37,8817-$ 8824.

(3) Krueger-Koplin, R. D.; Sorgen, P. L.; Krueger-Koplin, S. T.; Rivera-Torres, I. O.; Cahill, S. M.; Hicks, D. B.; Grinius, L.; Krulwich, T. A.; Girvin, M. E. J Biomol NMR 2004, 28, 43-57. 